\title{
Implementation and Complexity evaluation of Packet Combining for Multi-Code Transmission over Multi-Antenna Broadband Channel
}

\author{
Houda Chafnaji ${ }^{\ddagger *}$,Tarik Ait-Idir ${ }^{\ddagger *}$, and Samir Saoudi* \\ ${ }^{\ddagger}$ Communications Systems Department, INPT, Madinat Al Irfane, Rabat, Morocco \\ *Signal and Communications Department, INSTITUT TELECOM/ TELECOM Bretagne, CS 83818, 29238 Brest Cedex, France. \\ Email: houda.chafnaji@telecom-bretagne.eu, aitidir@ieee.org, samir.saoudi@telecom-bretagne.eu
}

\begin{abstract}
In this paper, a very low complexity packet combining approach for single user multi-code CDMA transmission over a broadband multiple input multiple output (MIMO) channel is presented. This new approach involves a simple modification to the traditional packet combining algorithms by introducing new variables recursively computed. This modification leads to a computational complexity as well as buffer size insensitive to retransmission number. Two packet combining techniques are introduced. The first technique performs packet combining jointly with turbo equalization, the second one performs the combination jointly with demapping.
\end{abstract}

Index Terms-Multi-code CDMA, broadband MIMO, Packet combining, frequency domain turbo equalization, integrated equalization, integrated demapping.

\section{INTRODUCTION}

Multi-code CDMA is a simple technique for supporting users with heterogeneous data rates [1], i.e. a user who wishes to transmit at higher data rate is simply assigned additional orthogonal walsh codes. Combined with MIMO multiplexing techniques, Multi-code CDMA systems have the ability to provide very high spectral efficiency through the transmission of different parallel substreams over multiple antennas [2], [3]. Propagation through multipath fading channel destroys the orthogonality between different spreading codes and introduces intersymbols interferences (ISI). Several authors studied time domain minimum mean square error based chip equalizer (MMSE-TDE) as an efficient technique to restore the spreading code orthogonality, so as to suppress the ISI [4], [5], [6]. However, the MMSE-TDE, which implements a sliding-window detection approach, involves inversion of large matrices, the receiver complexity is generally cubic in terms of the MMSE filter length. The Frequency Domain MMSE-based chip equalizer (MMSE-FDE) proposed in [7], has the main advantage of low complexity compared with MMSE-TDE. The authors in [7] combine the Cyclic Prefix (CP) concept with single-carrier multi-code CDMA to enable efficient frequency domain equalization of severe multi-paths fading channels.

In wireless networks, hybrid automatic repeat request $(\mathrm{H}-$ ARQ) combined with forward error correction (FEC) is considered as an important mechanism to ensure highly reliable communication. Several packet combining strategies have been developed to exploit the diversity among all transmissions [8], [9]. Recently, more attention has been paid to packet combining schemes for MIMO systems. In
[10], Onggosanusi et al. introduced symbol-level transmission combining using Zero Forcing (ZF) and MMSE detection techniques for MIMO flat fading channel. A packet combining technique with integrated equalization was initially introduced by Samra and Ding for single antenna systems using a maximum a-posteriori (MAP) equalizer [11]. The key idea is to perform equalization jointly with packet combining by considering each retransmission as an additional receive antenna. In [12], packet combining technique with integrated equalization was presented for MIMO systems using MMSETDE and operating over a fast fading channel. However, this receiver is mainly limited by the inversion of $N_{T}$ matrices of size $k N_{R} L_{F} \times k N_{R} L_{F}$ at the $k$ th transmission, where $N_{T}$ is the number of transmit antennas, $N_{R}$ is the number of receive antennas and $L_{F}$ is the MMSE filter length. For multi-transmission multi-code CDMA systems, the integrated equalization is performed in frequency domain to reduce the receiver complexity [13], [14].

This paper describes a very low complexity packet combining approach for ARQ with single user multi-code CDMA transmission link operating over a broadband MIMO channel. The proposed packet combining techniques are designed for turbo equalized system using frequency domain soft interference cancellation and MMSE filtering. We focus on the implementation and the complexity evaluation of two packet combining techniques. The first technique performs packet combining jointly with turbo equalization, the second one performs the combination jointly with demapper. The key idea of the proposed packet combining approach is to exploit the diversity among all transmissions with a very low cost by introducing new variables recursively computed. In the proposed approach, as the number of transmission increase, the memory requirement remains the same and the integrated equalization or demapping's computational complexity remains nearly unchanged. This paper is organized as follow: Section II presents the multi-transmission multi-code CPCDMA communication model. In section III, multi-antenna CP-CDMA Conventional receiver with no packet combining is presented as a comparative reference for the proposed packet combining approach in term of implementation and complexity evaluation. Section IV describes the proposed combining techniques with the new packet combining approach. The performance and complexity evaluation is presented in section $\mathrm{V}$. The paper is concluded in section VI.

Notations 
- $\operatorname{diag}\{\mathbf{x}\}$ and $\operatorname{diag}\left\{\mathbf{X}_{1}, \cdots, \mathbf{X}_{m}\right\}$ denote the diagonal matrix and the block diagonal matrix constructed from $\mathbf{x} \in \mathbb{C}^{n}$ and from $\mathbf{X}_{1}, \cdots, \mathbf{X}_{m} \in \mathbb{C}^{n_{1} \times n_{2}}$, respectively.

- $(.)^{T}$ and $(.)^{H}$ are the transpose and the transpose conjugate of the argument, respectively.

- $\mathbf{U}_{T}$ is a unitary $T \times T$ matrix whose $(m, n)$ th element is $\left(\mathbf{U}_{T}\right)_{m, n}=\frac{1}{\sqrt{T}} e^{-j(2 \pi m n / T)}$, and $j=\sqrt{-1}$.

- $\mathbf{U}_{T, N}=\mathbf{U}_{T} \otimes \mathbf{I}_{N}$, where $\mathbf{I}_{N}$ is the $N \times N$ identity matrix and $\otimes$ denotes the Kronecker product.

- For $\mathbf{x} \in \mathbb{C}^{T N}, \mathbf{x}_{f}$ denotes the Fourier transform of $\mathbf{x}$, i.e. $\mathbf{x}_{f}=\mathbf{U}_{T, N} \mathbf{x}$.

\section{MUlti-TRANSMisSiON CP-CDMA COMMUNICATION MODEL}

In this paper, we consider a point to point single user multicode CP-CDMA system operating over a broadband MIMO channel and employing an ARQ scheme at the upper layer with a maximum number of transmissions equal to $K$. At the transmitter side, an information block is coded using a $\rho$ rate encoder, interleaved with the aid of a random interleaver $\Pi$, spatially multiplexed over $N_{T}$ transmit antennas (index $t$ ), then symbol mapped to a constellation $\mathcal{S}$. The per-antenna symbol block is passed through a serial-to-parallel converter followed by a spreading module. Note that the same spreading matrix $\mathbf{W}=\left[\mathbf{w}_{1}, \cdots, \mathbf{w}_{C}\right]$ is used for each antenna, where $\mathbf{w}_{n}=\left[w_{1, n}, \cdots, w_{N, n}\right]$ is a walsh code of length $N$ and $C \leq N$ is the number of codes assigned to the user. The spreading sequence is normalized to $\left\|\mathbf{w}_{n}\right\|=1$ and all $N$ chips have equal energy. The $C$ parallel chip streams are then summed. The chip block at the output of the system is given by $\mathbf{x}=\left[\mathbf{x}_{0}^{T}, \cdots, \mathbf{x}_{T-1}^{T}\right]^{T}$, where $T$ denotes the per antenna chip block length (index $i$ ), $\mathbf{x}_{i}=\left[x_{1, i}, \cdots, x_{N_{T}, i}\right]^{T}$ and $x_{t, i}$ is expressed as,

$$
x_{t, i}=\sum_{n=1}^{C} s_{t, n, j} w_{i, n},
$$

where $s_{t, n, j}$ is the $j$ th symbol spread using the $n$th Walsh code and transmitted over the $t$ th antenna. Before transmission, the cyclic prefix is inserted.

The broadband MIMO channel is composed of a maximum number of chip spaced taps equal to $L$. The CP length is $T_{C P}(\geq L)$. Initially, a $N_{T} \times\left(T+T_{C P}\right)$ chips matrix is transmitted. Upon reception of a negative acknowledgment (NACK) message from the receiver, the chip matrix is retransmitted using the same coding, interleaving, modulation and spreading scheme. The MIMO channel is supposed to remain constant during one frame, and to independently change from one transmission to another. Let $\mathbf{H}_{0}^{(k)}, \cdots, \mathbf{H}_{L_{k}-1}^{(k)}$ denote the $N_{R} \times N_{T}$ matrices corresponding to the $L_{k} \leq L$ taps at transmission $k$, and which are made of zero-mean circularly symmetric complex Gaussian random variables verifying the following normalization condition,

$$
\mathbb{E}\left[\operatorname{diag}\left\{\sum_{l=0}^{L_{k}-1} \mathbf{H}_{l}^{(k)} \mathbf{H}_{l}^{(k)^{H}}\right\}\right]=N_{T} \mathbf{I}_{N_{R}} .
$$

We suppose that no knowledge of channel state information (CSI) is available at the transmitter side, and perfect channel

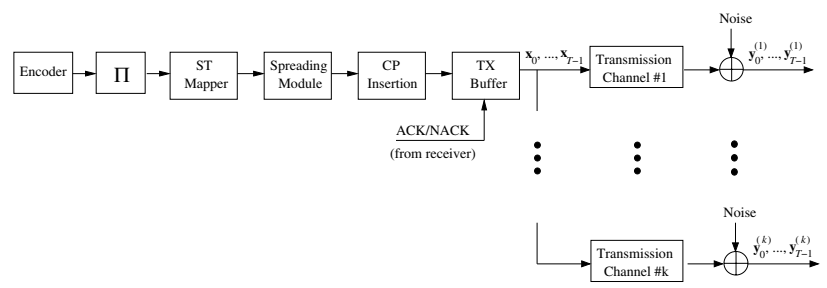

Fig. 1. Multi-code CP-CDMA transmitter with ARQ over time-varying MIMO-ISI communication channel

estimation is performed in reception. Equal power transmission strategy among the $N_{T}$ transmit antennas is therefore assumed,

$$
\mathbb{E}\left[\mathbf{x}_{i} \mathbf{x}_{i}^{H}\right]=\mathbf{I}_{N_{T}} .
$$

After the CP removal, the $N_{R} \times 1$ received signal at the $k$ th transmission and at discrete time $i$ is expressed as,

$$
\mathbf{y}_{i}^{(k)}=\sum_{l=0}^{L_{k}-1} \mathbf{H}_{l}^{(k)} \mathbf{x}_{(i-l) \bmod T}+\mathbf{n}_{i}^{(k)},
$$

where $\mathbf{n}_{i}^{(k)} \sim \mathcal{N}\left(\mathbf{0}_{N_{R} \times 1}, \sigma^{2} \mathbf{I}_{N_{R}}\right)$ is the thermal noise at the receiver side. The block communication model, at transmission $k$, can be written as,

$$
\mathbf{y}^{(k)}=\mathcal{H}_{c}^{(k)} \mathbf{x}+\mathbf{n}^{(k)}
$$

where $\mathbf{y}^{(k)} \triangleq\left[\mathbf{y}_{0}^{(k)^{T}}, \cdots, \mathbf{y}_{T-1}^{(k)^{T}}\right]^{T}, \quad \mathbf{n}^{(k)}=$ $\left[\mathbf{n}_{0}^{(k)^{T}}, \cdots, \mathbf{n}_{T-1}^{(k)^{T}}\right]^{T}$ and $\mathcal{H}_{c}^{(k)} \in \mathbb{C}^{T N_{R} \times T N_{T}}$ is a block circulant matrix whose first $T N_{R} \times N_{T}$ column matrix is $\left[\mathbf{H}_{0}^{(k)^{T}}, \cdots, \mathbf{H}_{L_{k}-1}^{(k)^{T}}, \mathbf{0}_{N_{T} \times\left(T-L_{k}\right) N_{R}}\right]^{T}$. Fig. 1 depicts the considered multi-code CP-CDMA scheme with multiple retransmissions over different multi-antenna channels.

As $\mathcal{H}_{c}^{(k)}$ is block circulant, it can be block diagonalized in a Fourier basis as $\mathcal{H}_{c}^{(k)}=\mathbf{U}_{T, N_{R}}^{H} \Lambda^{(k)} \mathbf{U}_{T, N_{T}}$, where

$$
\left\{\begin{array}{l}
\boldsymbol{\Lambda}^{(k)} \triangleq \operatorname{diag}\left\{\boldsymbol{\Lambda}_{0}^{(k)}, \cdots, \boldsymbol{\Lambda}_{T-1}^{(k)}\right\}, \\
\boldsymbol{\Lambda}_{i}^{(k)} \triangleq \sum_{l=0}^{L_{k}-1} \mathbf{H}_{l}^{(k)} e^{-j(2 \pi i l / T)} .
\end{array}\right.
$$

A discrete Fourier transform (DFT) is then applied to the received vector $\mathbf{y}^{(k)}$. The frequency domain communication model at $k$ transmissions is expressed as,

$$
\mathbf{y}_{f}^{(k)}=\boldsymbol{\Lambda}^{(k)} \mathbf{x}_{f}+\mathbf{n}_{f}^{(k)} .
$$

\section{Multi-Antenna Multi-Code CP-CDMA CONVENTIONAL RECEIVER WITH FREQUENCY DOMAIN MMSE-BASED TURBO EQUALIZATION}

The conventional receiver for multi-antenna multi-code $\mathrm{CP}$ CDMA, presented in this subsection, makes use of ARQ principle with no packet combiner at the receiver side. At transmission $k$, the receiver performs soft equalization and computes the extrinsic Log-Likelihood Ratio (LLR) about coded and interleaved bits with the aid of the communication model (7), and the a-priori information generated by the soft input soft output (SISO) decoder at the previous iteration. Interference cancellation is performed starting from the first iteration. Actually, the conventional receiver presented in this 
paper makes use of prior LLRs of coded and interleaved bits generated by the SISO decoder during the last iteration of previous transmission $k-1$. This idea was initially introduced by Narayanan and Stuber in the context of single antenna coded systems with ARQ [15].

First, soft inter-chip interference (ICI) (and also the intersymbol interference (ISI), in the case of $L \geq N+1$ ) is canceled from the received signal vector $\mathbf{y}_{f}^{(k)}$. Then, the resulting soft ICI/ISI-free signal enters an unconditional MMSE filter. Let $\tilde{\mathbf{x}}$ denotes the conditional estimate on $\mathbf{x}$ and $\sigma_{t, i}^{2}$ denotes the conditional variance of $x_{t, i}$. As presented in [16], the soft interferences cancellation and MMSE filtering can be implemented in the frequency domain using a forward and a backward filters. The MMSE estimate $\mathbf{z}_{f}^{(k)}$ on $\mathbf{x}_{f}$ at transmission $k$ is expressed as ,

$$
\mathbf{z}_{f}^{(k)}=\boldsymbol{\Phi}^{(k)} \mathbf{y}_{f}^{(k)}-\boldsymbol{\Psi}^{(k)} \tilde{\mathbf{x}}_{f},
$$

where $\boldsymbol{\Phi}^{(k)}=\operatorname{diag}\left\{\boldsymbol{\Phi}_{0}^{(k)}, \cdots, \boldsymbol{\Phi}_{T-1}^{(k)}\right\}$ is the forward filter given by,

$$
\left\{\begin{array}{l}
\boldsymbol{\Phi}_{i}^{(k)} \triangleq \frac{1}{\sigma^{2}}\left\{\mathbf{I}_{N_{T}}-\mathbf{D}_{i}^{(k)} \mathbf{C}_{i}^{(k)^{-1}}\right\} \boldsymbol{\Lambda}_{i}^{(k)^{H}} \\
\mathbf{C}_{i}^{(k)}=\sigma^{2} \tilde{\boldsymbol{\Xi}}^{-1}+\mathbf{D}_{i}^{(k)}
\end{array}\right.
$$

where $\mathbf{D}_{i}^{(k)}=\boldsymbol{\Lambda}_{i}^{(k)^{H}} \boldsymbol{\Lambda}_{i}^{(k)}$ and $\tilde{\boldsymbol{\Xi}}$ is an unconditional covariance matrix computed as the time average of conditional covariance matrices $\boldsymbol{\Xi}_{i}$ defined as,

$$
\boldsymbol{\Xi}_{i} \triangleq \operatorname{diag}\left\{\sigma_{1, i}^{2}, \cdots, \sigma_{N_{T}, i}^{2}\right\}
$$

and $\boldsymbol{\Psi}^{(k)}=\operatorname{diag}\left\{\boldsymbol{\Psi}_{0}^{(k)}, \cdots, \boldsymbol{\Psi}_{T-1}^{(k)}\right\}$ is the backward filter given by,

$$
\left\{\begin{array}{l}
\boldsymbol{\Psi}_{i}^{(k)} \triangleq \boldsymbol{\Phi}_{i}^{(k)} \boldsymbol{\Lambda}_{i}^{(k)}-\boldsymbol{\Upsilon}^{(k)} \\
\boldsymbol{\Upsilon}^{(k)}=\frac{1}{T} \sum_{i=0}^{T-1} \boldsymbol{\Phi}_{i}^{(k)} \boldsymbol{\Lambda}_{i}^{(k)}
\end{array}\right.
$$

After computing (8), the inverse DFT (IDFT) is then applied to $\mathbf{z}_{f}^{(k)}$ to obtain the equalized time domain chip sequence,

$$
\mathbf{z}^{(k)}=\mathbf{U}_{T, N_{T}}^{H} \mathbf{z}_{f}^{(k)} .
$$

the MMSE estimate $z_{t, i}^{(k)}$ corresponding to antenna $t$ and channel use $i$ after $k$ transmission can be simply extracted from $\mathbf{z}^{(k)}$ as $z_{t, i}^{(k)}=\mathbf{e}_{t, i}^{H} \mathbf{z}^{(k)}$, with $\mathbf{e}_{t, i}$ denotes the $\left(N_{T} i+t\right)$ th vector of the canonical basis. After despreading, the extrinsic LLRs value $\phi_{t, j, m}^{(e)}$ corresponding to coded and interleaved bit $b_{t, j, m}$ are then produced by the demapper using the max-log simplification,

$$
\begin{aligned}
\phi_{t, j, m}^{(e)}= & \min _{s \in \mathcal{S}_{0}^{m}}\left\{\boldsymbol{\xi}_{t, j}^{(k)}(s)-\sum_{m^{\prime} \neq m} \phi_{t, j, m^{\prime}}^{(a)} \lambda_{m^{\prime}}\{s\}\right\} \\
& -\min _{s \in \mathcal{S}_{1}^{m}}\left\{\boldsymbol{\xi}_{t, j}^{(k)}(s)-\sum_{m^{\prime} \neq m} \phi_{t, j, m^{\prime}}^{(a)} \lambda_{m^{\prime}}\{s\}\right\},
\end{aligned}
$$

with

$$
\boldsymbol{\xi}_{t, j}^{(k)}(s)=\frac{\left|r_{t, j}^{(k)}-g_{t, j}^{(k)} s\right|^{2}}{\theta_{t, j}^{(k)^{2}}},
$$

where $r_{t, j}^{(k)}, g_{t, j}^{(k)}$ and $\theta_{t, j}^{(k)}$ denote, respectively, the despreading module output, the equivalent channel gain at the output of equalizer and the residual interference variance corresponding to discrete time $j$, transmit antenna $t$ and transmission index $k$. $\phi_{t, j, m^{\prime}}^{(a)}$ is the a-priori LLR for coded bit $b_{t, j, m^{\prime}}$ obtained from the decoder in the previous iteration, $\lambda_{m^{\prime}}\{s\}$ is an operator extracting the $m^{\prime}$ th bit labeling the symbol $s \in \mathcal{S}$ and $\mathcal{S}_{\beta}^{m}$ is the set of symbols having the $m$ th bit set to $\beta$, i.e. $\mathcal{S}_{\beta}^{m}=$ $\left\{s: \lambda_{m}\{s\}=\beta\right\}$. The LLRs calculated by the demapper is then desinterleaved and fed to a SISO decoder.

\section{LOW-COMPlexity PACKet COMBINING WiTH SPACE-TIME FREQUENCY DOMAIN TURBo EQUALIZATION}

In this section, two low complexity packet combining techniques are introduced. The first one performs the packet combining jointly with frequency domain MMSE-based equalization. The second one performs it jointly with demapping.

\section{A. Frequency Domain Packet Combining with Integrated MMSE-Based Turbo Equalization}

A multi-antenna multi-code CDMA system with ARQ introduces more spatial and temporal diversities as the same block is transmitted over different and independent MIMO channels. A diversity gain can be achieved by using a joint MMSE-FDE combiner. The idea of joint combiner presented in [13], [14] consists in performing packet combining jointly with frequency domain equalization by considering each transmission as an additional set of virtual receive antennas. As the equalization is applied to an equivalent system of $k N_{R}$ receive antennas, the problem dimensionality grows linearly with the number of transmissions. To cope with this issue, we introduce a simple modification to the MMSEFDE algorithm, presented in section III, to allow packet combining at the equalization level without any increase in the problem dimensionality. In contrast with [13], [14], the proposed algorithm remains the same from one transmission to another and the equalization is performed in a real system size with $N_{R}$ receive antennas. For this purpose, we introduce two new variables $\underline{\tilde{\mathbf{y}}}_{f}^{(k)} \in \mathbb{C}^{T N_{T} \times 1}$ and $\underline{\mathbf{D}}^{(k)} \in \mathbb{C}^{T N_{T} \times T N_{T}}$ recursively computed.

The proposed joint MMSE-FDE is described as follow. At transmission $k$, the received signal $\mathbf{y}^{(k)}$ is passed through a DFT module. The frequency domain receive signal $\mathbf{y}_{f}^{(k)}$ is multiplied by frequency domain channel matrix $\Lambda^{(k)}$ as,

$$
\tilde{\mathbf{y}}_{f}^{(k)}=\boldsymbol{\Lambda}^{(k)^{H}} \mathbf{y}_{f}^{(k)} \in \mathbb{C}^{T N_{T} \times 1} .
$$

To take advantage of joint equalization combining without any increase in the problem dimensionality, the different retransmissions are combined as,

$$
\underline{\tilde{\mathbf{y}}}_{f}^{(k)}=\sum_{u=1}^{k} \tilde{\mathbf{y}}_{f}^{(u)}
$$


A buffer of size $T N_{T}$ is introduced to store the vector $\underline{\tilde{\mathbf{y}}}_{f}^{(k)}$. So at each transmission $k, \underline{\mathbf{y}}_{f}^{(k)}$ can be updated using the recursion,

$$
\underline{\tilde{\mathbf{y}}}_{f}^{(k)}=\tilde{\mathbf{y}}_{f}^{(k-1)}+\tilde{\mathbf{y}}_{f}^{(k)},
$$

Furthermore, to calculate forward and backward filters, the joint MMSE-FDE uses the current transmission channel matrix as well as the last retransmissions ones. Instead of using a buffer of size $T(K-1) N_{R} N_{T}$ to store the channel matrices $\boldsymbol{\Lambda}^{(1)}, \cdots, \boldsymbol{\Lambda}^{(K-1)}$ of transmissions $1, \cdots, K-1$, we introduce a buffer of size $T N_{T}^{2}$ to store $\underline{\mathbf{D}}^{(k)}=\sum_{u=1}^{k} \mathbf{D}^{(u)}$ with $\mathbf{D}^{(u)}=\boldsymbol{\Lambda}^{(u)^{H}} \boldsymbol{\Lambda}^{(u)}$. At each transmission $k, \underline{\mathbf{D}}^{(k)}$ can be then updated using the recursion,

$$
\underline{\mathbf{D}}^{(k)}=\underline{\mathbf{D}}^{(k-1)}+\mathbf{D}^{(k)} .
$$

Once the variables in (15) and (16) are updated the $k$ th transmission can be discarded. The joint MMSE-FDE can be implemented by a low complexity forward and backward filters as follow,

$$
\mathbf{z}_{f}^{(k)}=\boldsymbol{\Gamma}^{(k)} \underline{\tilde{\mathbf{y}}}_{f}^{(k)}-\boldsymbol{\Omega}^{(k)} \tilde{\mathbf{x}}_{f},
$$

where $\boldsymbol{\Gamma}^{(k)}=\operatorname{diag}\left\{\boldsymbol{\Gamma}_{0}^{(k)}, \cdots, \boldsymbol{\Gamma}_{T-1}^{(k)}\right\}$ is the forward filter given by,

$$
\left\{\begin{array}{l}
\boldsymbol{\Gamma}_{i}^{(k)} \triangleq \frac{1}{\sigma^{2}}\left\{\mathbf{I}_{N_{T}}-\underline{\mathbf{D}}_{i}^{(k)} \mathbf{C}_{i}^{(k)^{-1}}\right\} \\
\mathbf{C}_{i}^{(k)}=\sigma^{2} \tilde{\boldsymbol{\Xi}}^{-1}+\underline{\mathbf{D}}_{i}^{(k)},
\end{array}\right.
$$

and $\boldsymbol{\Omega}^{(k)}=\operatorname{diag}\left\{\boldsymbol{\Omega}_{0}^{(k)}, \cdots, \boldsymbol{\Omega}_{T-1}^{(k)}\right\}$ is the backward filter given by,

$$
\left\{\begin{array}{l}
\boldsymbol{\Omega}_{i}^{(k)} \triangleq \boldsymbol{\Gamma}_{i}^{(k)} \underline{\mathbf{D}}_{i}^{(k)}-\boldsymbol{\Upsilon}^{(k)} \\
\boldsymbol{\Upsilon}^{(k)}=\frac{1}{T} \sum_{i=0}^{T-1} \boldsymbol{\Gamma}_{i}^{(k)} \underline{\mathbf{D}}_{i}^{(k)}
\end{array}\right.
$$

As $\underline{\mathbf{D}}^{(k)}$ is updated recursively, the computation complexity of filters in (18) and (19) is insensitive to the number of retransmissions.

After computing (17), the inverse DFT (IDFT) is then applied to $\mathbf{z}_{f}^{(k)}$ to obtain the equalized time domain chip sequence. After despreading, the extrinsic LLRs is computed using (13). The output of the demapper is then desinterleaved and fed to a SISO decoder. The block diagram of the proposed iterative ARQ receiver with integrated equalization at the $k$ th transmission is presented in Fig. 2.

\section{B. Packet Combining with integrated demapping}

In contrast with joint MMSE-FDE receiver, the joint demapping receiver performs space-time frequency domain equalization separately for each transmission and combines the different packets at the demapper level. At each transmission $k$, the Soft ICI/ISI cancellation and MMSE filtering are performed similarly to (8). Assuming independence between despreading module outputs $r_{t, j}^{(1)}, \cdots, r_{t, j}^{(k)}$, the different retransmissions can be combined recursively as,

$$
\underline{\boldsymbol{\xi}}_{t, j}^{(k)}(s)=\underline{\boldsymbol{\xi}}_{t, j}^{(k-1)}(s)+\boldsymbol{\xi}_{t, j}^{(k)}(s)
$$

For this purpose we introduce a Buffer of size $T N_{T} 2^{|\mathcal{S}|}$ to store $\underline{\boldsymbol{\xi}}_{t, j}^{(k)}(s)$. The extrinsic LLRs value $\phi_{t, j, m}^{(e)}$ corresponding to coded and interleaved bit $b_{t, j, m}$ are then calculated as,

$$
\begin{aligned}
\phi_{t, j, m}^{(e)}= & \min _{s \in \mathcal{S}_{0}^{m}}\left\{\underline{\boldsymbol{\xi}}_{t, j}^{(k)}(s)-\sum_{m^{\prime} \neq m} \phi_{t, j, m^{\prime}}^{(a)} \lambda_{m^{\prime}}\{s\}\right\} \\
& -\min _{s \in \mathcal{S}_{1}^{m}}\left\{\underline{\boldsymbol{\xi}}_{t, j}^{(k)}(s)-\sum_{m^{\prime} \neq m} \phi_{t, j, m^{\prime}}^{(a)} \lambda_{m^{\prime}}\{s\}\right\},
\end{aligned}
$$

Block diagram of the proposed receiver is presented in Fig. 3 .

\section{Complexity Evaluation}

Herein, we briefly present the proposed packet combining techniques implementation cost. For joint MMSE-FDE, the implementation cost depends only on the number of transmit antennas $N_{T}$ and the per antenna chip block length $T$. For joint demapping, the implementation cost depends on the same parameters and also on the constellation length. The memory and the additive computational complexity requirements for proposed packet combining techniques are resumed in table I.

\begin{tabular}{|c||c||c|}
\hline & Joint MMSE-FDE & Joint demapping \\
\hline \hline Memory & $T N_{T}\left(1+N_{T}\right)$ & $T N_{T} 2^{|\mathcal{S}|}$ \\
\hline \hline Additions & $T N_{T}\left(1+N_{T}\right)$ & $T N_{T} 2^{|\mathcal{S}|}$ \\
\hline
\end{tabular}

TABLE I

THE MEMORY AND THE ADDITIVE COMPUTATIONAL COMPLEXITY REQUIREMENTS

Note that the implementation of the proposed packet combining schemes in the conventional receiver involves a memory size and an additive computational complexity insensitive to the number of transmissions, which is very attractive particularly for mobile receivers.

\section{Numerical Results}

In this section, the performance and the complexity of the proposed multi-antenna multi-code CP-CDMA receivers are evaluated. The system used for the evaluation has $N_{T}=$ $\{2,4\}$ transmit antennas, $N_{R}=\{1,2,4\}$ receive antennas, spreading factor $N=16$ with all codes are assigned to one user $(C=16)$, Quadrature Phase Shift Keying (QPSK) modulation and 16 states convolutional encoder with polynomial generators $(35,23)_{8}$. The length of the coded frame is 2048 bits including tails. The MIMO channel has $L=10$ chip spaced paths with equally distributed power. The CP length is $T_{C P}=10$. We employ the Max-Log-MAP algorithm for SISO decoding. The iterative receivers run five turbo iterations for each transmission and the maximum number of transmissions is set to $K=3$. 


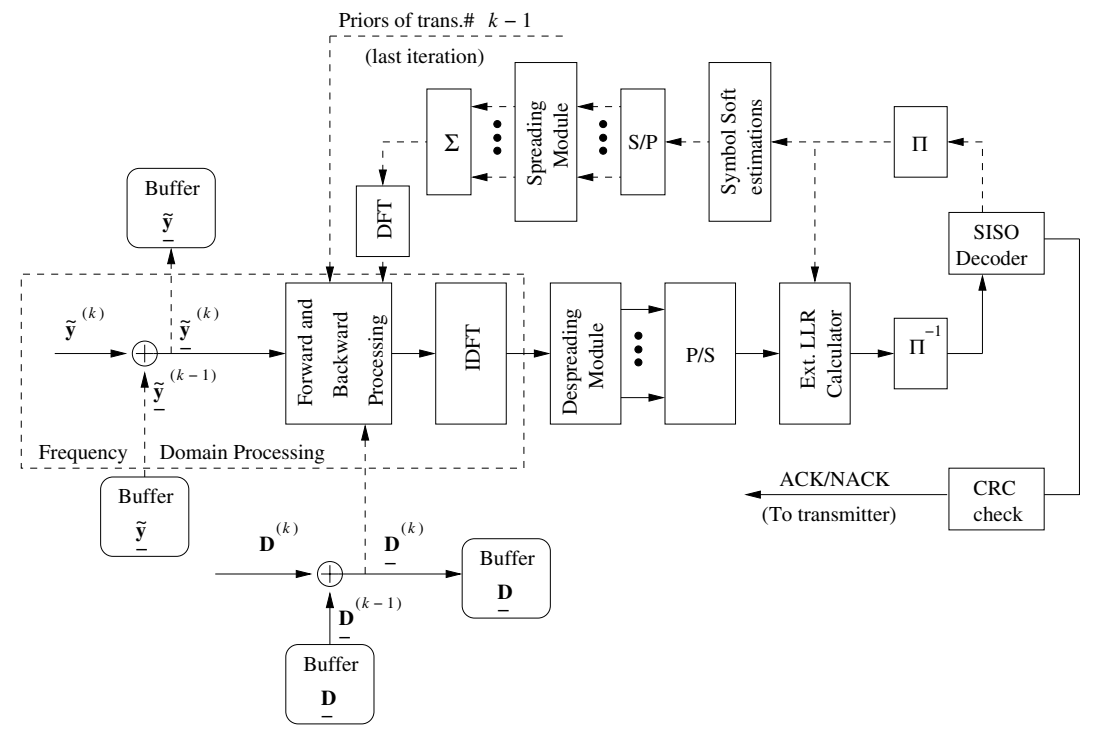

Fig. 2. Multi-antenna multi-code CP-CDMA receiver at $k$ th transmission with packet combining integrated frequency domain MMSE-based chip level turbo equalization

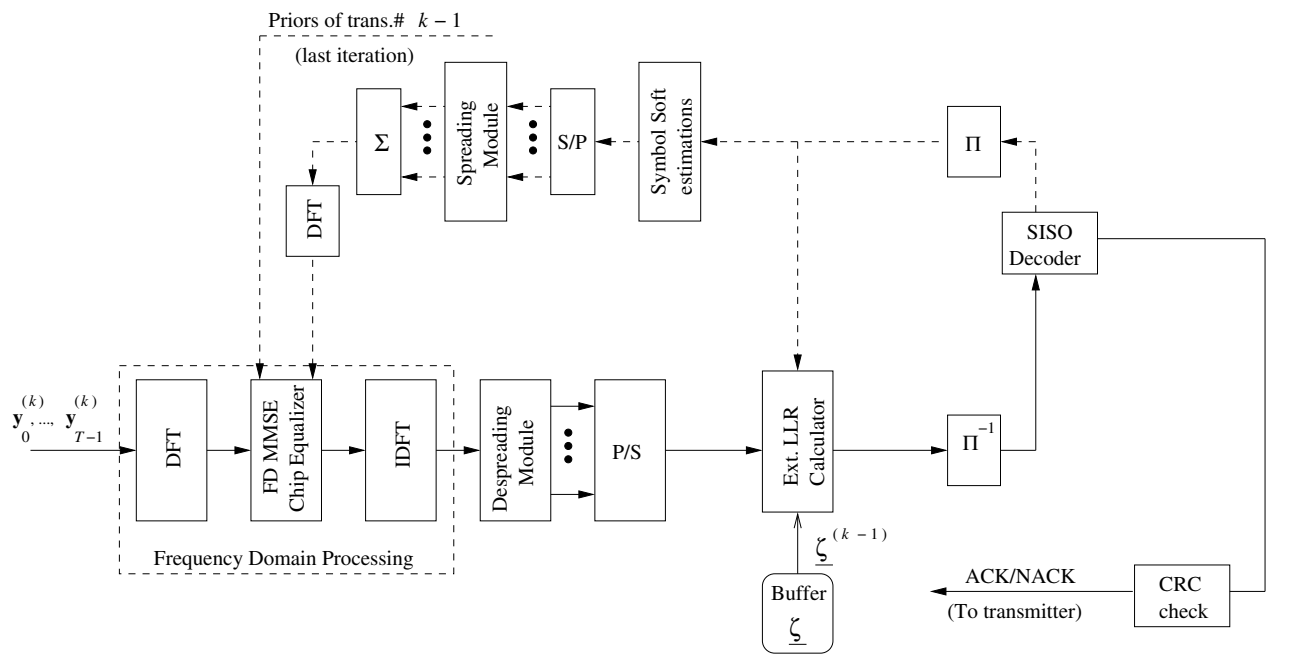

Fig. 3. Multi-antenna multi-code CP-CDMA receiver at $k$ th transmission with disjoint frequency domain MMSE-based chip level turbo equalization and joint demapping

We start by evaluating the complexity and the block error rate (BLER) performance of the considered system with $N_{T}=2$ and $N_{R}=2$. For this configuration, the joint MMSE-FDE implementation cost is less expensive than joint demapping one. Furthermore, we observe in Fig. 4 that the receiver with joint MMSE-FDE technique outperforms the one with joint demapping by $0.7 \mathrm{~dB}$ at $10^{-2}$ BLER for both second and third transmissions. Fig. 4 plots also the matched filter bound (MFB) to evaluate the diversity achievement of proposed algorithms. Thanks to joint equalization, a maximum of diversity is achieved and the gap between joint MMSE-FDE and MFB is reduced from $4 \mathrm{~dB}$ for the first transmission to $1 \mathrm{~dB}$ for the third transmission at $10^{-2}$ BLER.

We also evaluate the system configuration with large number of antennas $N_{T}=N_{R}=4$. In this case, the joint demapping is the less expensive technique. However, joint MMSE-FDE still the best in term of performance (Fig. 5).
As the performance gap between this two techniques is less than $1 \mathrm{~dB}$, the joint demapping can be the best candidate technique for system with large number of antennas and low level modulation.

Now we consider multiple input single output transmission systems which are of special interest for downlink radio mobile applications. Fig. 6 plots BLER performance for $N_{T}=2$ and $N_{R}=1$. Packet combining with integrated equalization scheme clearly outperforms joint demapping, the gap between this two techniques is more than $5 \mathrm{~dB}$ for the second transmission and $3 \mathrm{~dB}$ for the third transmission at $10^{-2}$ BLER.

\section{CONCLUSIONS}

In this paper, a very low complexity packet combining approach for ARQ with single user multi-code CDMA transmission link operating over a broadband MIMO channel 


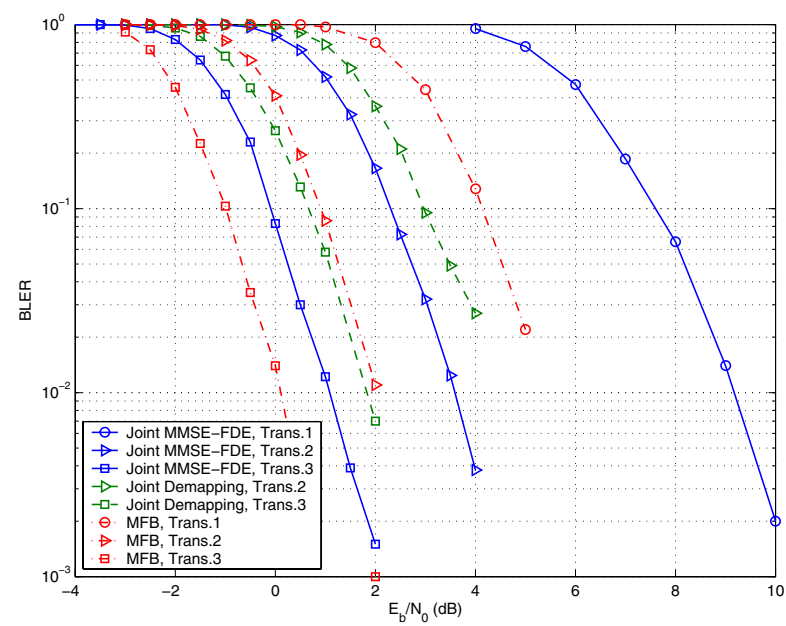

Fig. 4. BLER performance with $N_{T}=2, N_{R}=2, L=10$ equal power taps profile, spectrum efficiency $=32 \mathrm{bits} /$ channel use.

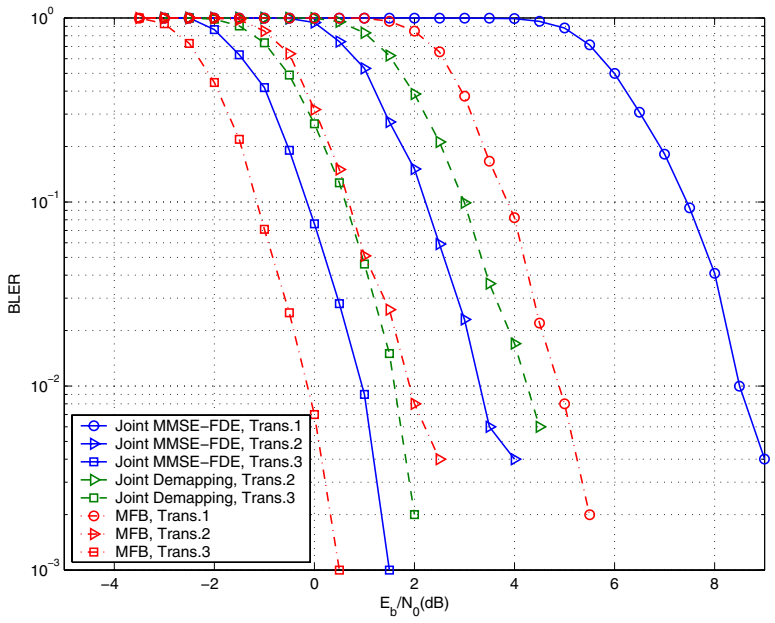

Fig. 5. BLER performance with $N_{T}=4, N_{R}=4, L=10$ equal power taps profile, spectrum efficiency $=64 \mathrm{bits} /$ channel use.

has been introduced. The key idea of this approach is to exploit the diversity among all transmissions with a very low cost by introducing new variables recursively computed. Two packet combining techniques are presented. the first technique consists in performing packet combining jointly with frequency domain chip level equalization. The second proposed technique performs packet combining jointly with demapping. Simulations have demonstrated that the packet combining scheme with integrated equalization clearly outperforms packet combining with integrated demapping especially for overloaded configurations. However, It has been shown that for system with large number of antennas and low level modulation, joint demapping is the most attractive technique in term of implementation cost with a very small loss in performance.

\section{REFERENCES}

[1] C.-L. I and R. D. Gitlin, "Multi-code CDMA wireless personal communications networks," in Proc. IEEE Int. Conf. Commun., Seattle, WA, June 1995, pp. 1060-1064.

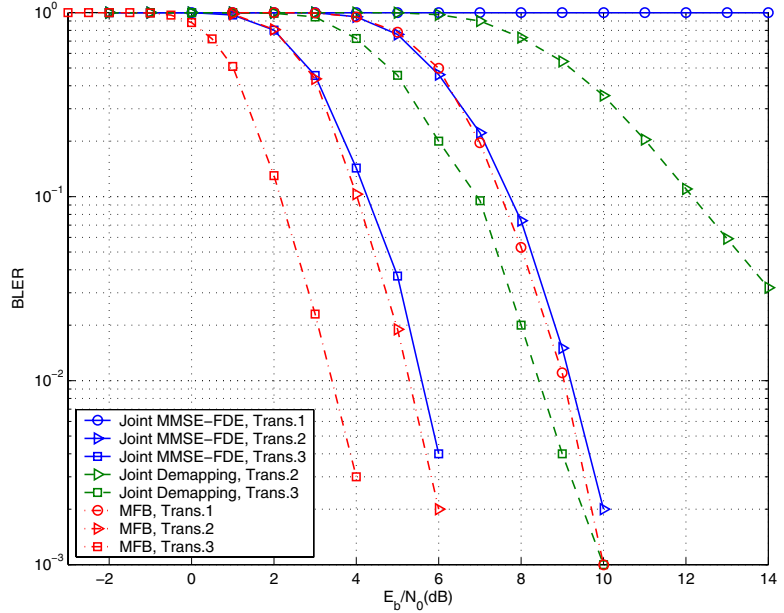

Fig. 6. BLER performance with $N_{T}=2, N_{R}=1, L=10$ equal power taps profile, spectrum efficiency $=32 \mathrm{bits} /$ channel use.

[2] P. W. Wolniansky, G. J. Foschini, and G. D. Valenzuela, "V-BLAST : An architecture for realizing very high data rates over the rich scattering wireless channel," in Proc. Int. Symp. Signals, Systems, Electron. , Pisa, Italy, Sep. 1998.

[3] H. Huang, H. Viswanathan, and G. J. Foschini, "Multiple antennas in cellular CDMA systems: Transmission, detection, and spectral efficiency," IEEE Trans. Wireless Commun., vol. 1, no. 4, pp. 383-392, Jul. 2002.

[4] U. Madhow and M. L. Honig, "MMSE Interference suppression for Direct-Sequence Spread-spectrum CDMA," IEEE Trans. Communications, vol. 12, no. 4, pp. 3178-3188, 1994.

[5] K. Hooli, M. J. Heikkila, P. Komulainen, M. Latva-aho, and J. Lilleberg, "Chip-lebel channel equalization in WCDMA downlink," EURASIP Journal on Applied Signal Processing, Aug.2002, pp. 757-770.

[6] J. Choi, "MMSE Equalization of Downlink CDMA Channel utilizing Unused Orthogonal Spreading Sequences," IEEE Trans. Signal Proc., vol.51, pp.1390-1402, May 2003.

[7] K. Baum, T. Thomas, F. Vook, and V. Nangia, "Cyclic-Prefix CDMA: An Improved Transmission Method for Broadband DS-CDMA Cellular Systems," IEEE WCNC 2002, Vol.1, pp. 183-188, March 2002.

[8] D. Chase, "Code combining-A maximum-likelihood decoding approach for combining an arbitrary number of noisy packets," IEEE Trans. Commun., vol. COM-33, no. 5, pp. 385-393, May 1985.

[9] B. Harvey and S. Wicker, "Packet combining systems based on the Viterbi decoder," IEEE Trans. Commun., vol. 42, no. 2-4, pp. 15441557, Feb.-Apr. 1994.

[10] E. N. Onggosanusi, A. G. Dabak, Y. Hui, and G. Jeong, "Hybrid ARQ transmission and combining for MIMO systems," in Proc. IEEE Int. Conf. Commun., vol. 5, May 2003, pp. 3205-3209.

[11] H. Samra and Z. Ding, "New MIMO ARQ protocols and joint detection via sphere decoding," IEEE Trans. Sig. Proc., vol. 54, no. 2, pp. 473482, Feb. 2006.

[12] T. Ait-Idir, H. Chafnaji, and S. Saoudi, "Joint hybrid ARQ and iterative space-time equalization for coded transmission over the MIMO-ISI channel," IEEE WCNC 2008, Las Vegas, NV, Mar-Apr. 2008.

[13] D. Garg and F. Adachi, "Packet Access Using DS-CDMA With Frequency-Domain Equalization ," IEEE J. Sel. Areas Commun., vol. 24 , no. 1, pp.161-170, Jan. 2006.

[14] H. Chafnaji, T. Ait-Idir, and S. Saoudi, "Packet Combining and Chip Level Frequency Domain Turbo Equalization for Multi-Code Transmission over Multi-Antenna Broadband Channel," IEEE PIMRC 2008, Cannes, Sept. 2008.

[15] K. Narayanan and G. Stuber, "A novel ARQ technique using the turbo coding principle," IEEE Commun. Lett., vol. 1, no. 3, pp. 49-51, Mar. 1997.

[16] R. Visoz, A. O. Berthet, and S. Chtourou, "Frequency-domain block turbo-equalization for single-carrier transmission over MIMO broadband wireless channel," IEEE Trans. Commun., vol. 54, no. 12, pp. 2144-2149, Dec. 2006. 